\title{
The Viennese EDDY Study as a Role Model for Obesity: Prevention by Means of Nutritional and Lifestyle Interventions
}

\author{
Kurt Widhalm ${ }^{a, b}$ Oliver Helk ${ }^{a}$ Otmar Pachinger ${ }^{c}$ \\ ${ }^{a}$ Austrian Institute for Clinial Nutrition, Vienna, Austria; b Medical University Vienna, Vienna, \\ Austria; ' Austrian Heart Foundation, Vienna, Austria
}

\section{Keywords}

Lifestyle change · Obesity · Prevention · School-based intervention

\begin{abstract}
Objectives: Obesity in children and adolescents is a worldwide dramatic health problem, for which treatment is mostly unsuccessful. Therefore, prevention is the most important measure to tackle this problem. The 'EDDY' study as an interventional cohort study with a 1-year lifestyle intervention aimed to affect the lifestyle and nutrition habits of adolescents by intervention with nutritional training and sports programs to prevent obesity. Methods: Four Viennese schools were cluster-randomized into an intervention group and a control group. A total of 141 pupils aged 11-14 years were included. The intervention group received a comprehensive, age-appropriate training on nutrition and lifestyle exercise intervention for 12 months. Before and after intervention and at two follow-ups, subjects were anthropometrically measured. In addition, knowledge of nutritional issues and eating habits were measured with questionnaires. Results: The data imply an improvement of nutrition knowledge, a significant reduction in the consumption of junk food $(p=0.01)$, sweets $(p=0.001)$ and salty snacks $(p<0.001)$ as well as a slight improvement of physical performance after intervention. Although there was a trend for a less increase of body fat in the intervention group, no significant changes could have been shown in the anthropometric data. Conclusions: An age-adjusted lifestyle intervention based on dietary training and exercise can improve the nutritional knowledge and eating habits of school children.

(C) 2018 The Author(s)

Published by S. Karger GmbH, Freiburg
\end{abstract}

Univ.-Prof. Dr. Kurt Widhalm

Head of Austrian Academic Institute for Clinical Nutrition

Alserstraße 14/4a

1090 Vienna, Austria

kwidhalm@gmx.at 
Widhalm et al.: The Viennese EDDY Study as a Role Model for Obesity: Prevention by Means of Nutritional and Lifestyle Interventions

\section{Introduction}

Overweight and obesity in children and adolescents are major health problems in Europe. Prevention is declared as a high priority scientific research aim by the World Health Organization (WHO).

Current projections from the WHO highlight the urgency of the timely implementation of effective prevention approaches [1]. A recently published worldwide survey pointed out the dramatic increase of the prevalence of obesity between 1975 and 2016 from 0.7 to $5.6 \%$ in girls and from 0.9 to $7.8 \%$ in boys [2]. Furthermore, data from the European HELENA study showed that between 19.5 and 26.4\% (females and males) of adolescents in Europe are overweight or obese [3].

To date, the effects of preventive measures upon overweight and risk factors for cardiovascular diseases in children and adolescents have been described in only a few controlled studies.

The 'EDDY' study as an evaluated prevention project with a 2-year lifestyle intervention was carried out at four Viennese schools. The aim was to assess the health and nutritional status as well as the nutrition habits of a representative sample of 147 Viennese pupils aged 11-14 years and to affect the lifestyle and nutrition habits of the subjects by intervention with nutritional training and sports programs.

\section{Subjects and Methods}

\section{Subjects}

141 subjects aged 11-14 years were recruited into the study. The pupils were recruited in two grammar schools and two middle schools in Vienna. The subjects were cluster-randomized into an intervention and a control group, with each school representing an individual cluster. A total of $n=82$ pupils (mean age 11.69 \pm 0.67 years) were assigned to the intervention group, $n=59$ pupils (mean age $12.04 \pm 0.59$ years) were assigned to the control group (table 1). Due to illness or absence at the times of measurements, not all data from the subjects were collected at all points of time. This led to small variations in the sample between the different test parameters.

A positive ethical opinion for the EDDY-Study was granted by the ethical committee of the City Vienna (EK_13_070_0413).

\section{Measurements}

Before and after each intervention as well as during follow-up measurements at 6 months and after the last intervention at 12 months, the subjects were anthropometrically measured, and the nutritional knowledge and behavior as well as the physical fitness were tested. In addition, blood samples were taken, the results of which will be discussed in another paper.

Weight, Height, and Body Composition

The height of the subjects was measured using a stadiometer. Weight and body fat percentage were determined by bioelectric impedance analysis (Tanita MC 980-MA; Tanita, Tokyo, Japan).

To classify the body fat percentage, the results were categorized into body fat percentiles for children and adolescents according to percentile distributions published by Mc Carthy et al. [4].

BMI standard deviation score were calculated according to Must and Anderson [5].

\section{Nutritional Knowledge}

The nutritional knowledge was measured by a specially designed quiz 'What do you know about nutrition?' based on 12 age-adjusted questions about foods and food ingredients [6].

\section{Food Preferences}

Food preferences were determined retrospectively using the Food Frequency Questionnaire for children and adolescents [7]. 
Widhalm et al.: The Viennese EDDY Study as a Role Model for Obesity: Prevention by Means of Nutritional and Lifestyle Interventions

Table 1. Distribution of the study population

\begin{tabular}{llll}
\hline & $\mathrm{n}$ & Male & Female \\
\hline Intervention group & 82 & 41 & 41 \\
Control group & 59 & 34 & 25 \\
\hline
\end{tabular}

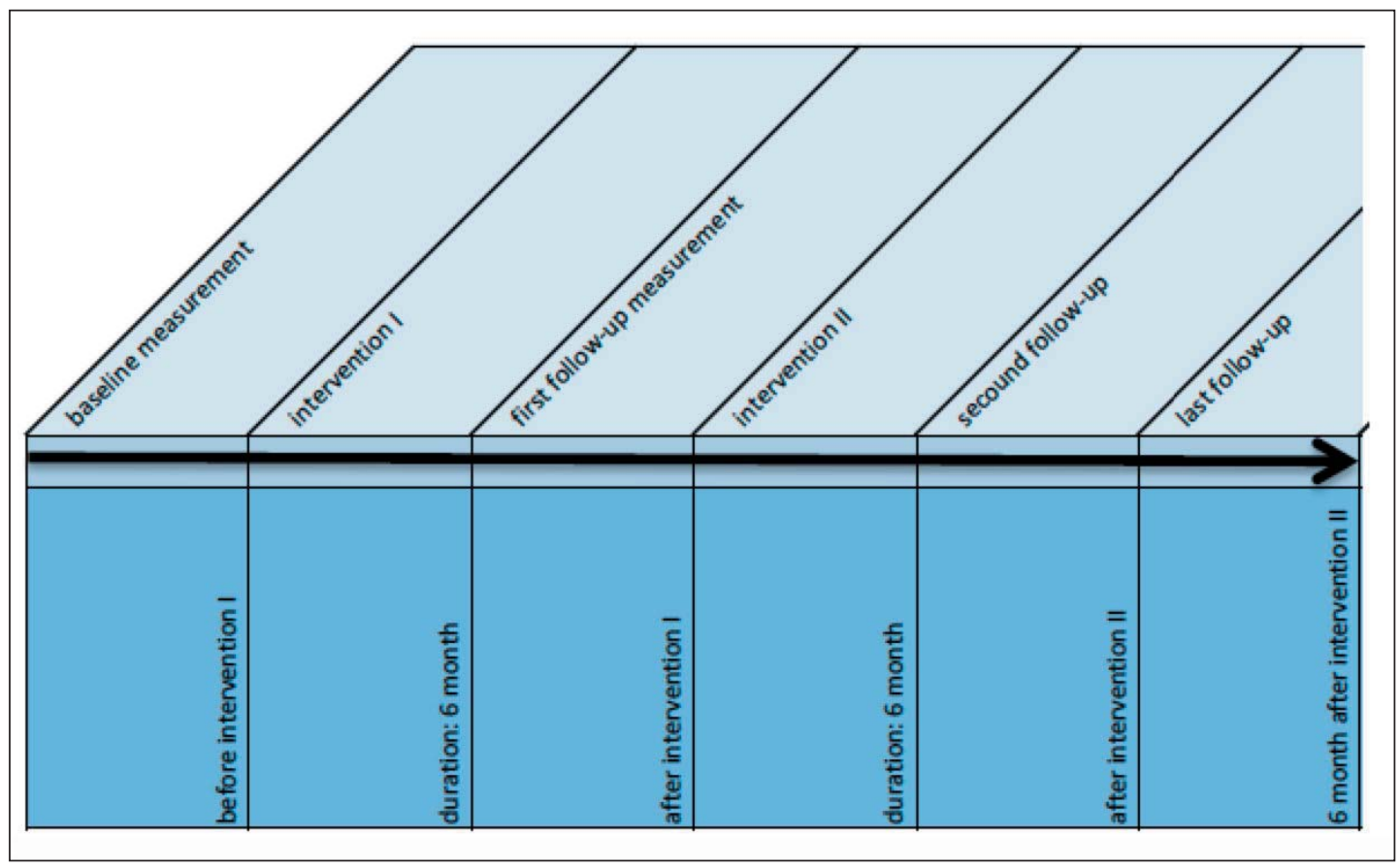

Fig. 1. Timeline of the project.

\section{Physical Fitness}

To collect the physical fitness the 'Deutsche Motorik Test 6-18' [8] was used which includes the following test items: 6-min endurance run, standing long jump, push-ups (40 s), sit-ups (40 s), $20 \mathrm{~m}$ sprint, reversed balance, skip back and forth sideways, and forward bend.

\section{Psychological Measurements}

The interdisciplinary test system 'AD-EVA' [9] was used to assess psychological as well as sports-related and nutrition-related factors influencing the development of overweight and obesity.

\section{Interventions}

The intervention group received $20 \mathrm{~h}$ of nutritional training, five lessons about physiology and the impact of a healthy lifestyle, and 20-hour sports and exercise intervention (fig. 1).

\section{Lifestyle Lessons}

At the beginning of the first intervention cycle the pupils received a 5-hour lifestyle intervention. During this intervention the adolescents acquired basic knowledge about physiological and biochemical processes in the human body.

The functions and tasks of the skeletal apparatus, the musculature, the adipose tissue, and metabolically relevant hormones as well as the regulation of the blood glucose level were discussed.

In addition, the health risks of smoking, alcohol consumption, and insufficient movement and sports were explained. 
Widhalm et al.: The Viennese EDDY Study as a Role Model for Obesity: Prevention by Means of Nutritional and Lifestyle Interventions

\section{Nutritional Lessons}

The training courses on 'healthy nutrition' were divided into two cycles. The pupils received a 10-hour nutritional training during each semester, which results in a intervention time of $20 \mathrm{~h}$ of nutritional training through the whole study.

In the first intervention cycle the theoretical basic concepts of dietetics were taught. The topics in this intervention cycle were:

- $\quad$ The relevance of macronutrients and micronutrients

- The importance of a regular food and liquid intake for daily performance

- $\quad$ The origin of foods

- $\quad$ The optimal dietary intake on the basis of the healthy eating pyramid

- The labeling of food packaging

- The right choice of beverages

- $\quad$ The right choice of meals and foods.

The lessons in the first intervention cycle were held as interactive presentations following short exercise on worksheets at the end of the presentation. The pupils received handouts with important information about the topics.

In a second intervention cycle the acquired knowledge was deepened by practical exercises and experiments. The subjects performed experiments in which they were able to taste different foods, visualize the water and fat content of different groceries, assign processed foods to their original raw materials, and prepare healthy snacks and meals together. In addition the pupils received a specifically designed 'EDDY Magazine' with instructions of the experiments and background information as well as further exercises for homework.

The experiments and exercises in the second intervention cycle were:

- $\quad$ Building a big healthy eating pyramid for the classroom

- Visualize hidden fat and water in groceries with a high amount of fat or water (e.g. cucumbers, water melon, croissant, peanut butter)

- Preparing a healthy snack with a selection of different ingredients

- $\quad$ Matching the proceeded groceries to their raw elements

- $\quad$ Origin, season, and ripe of fruits and vegetables.

- $\quad$ Cooking a healthy meal together

- $\quad$ Finding ten reasons to live healthy (as a resume of the intervention).

\section{Sports and Movement Lessons}

The pupils received a 10-hour sports and movement intervention per semester during their normal physical education lessons. Similar to the nutritional training, they received $20 \mathrm{~h}$ of sports and movement intervention during the whole study duration.

The sports interventions served to stimulate the subjects to integrate movement into their everyday life through movement and coordination games. In addition, the exercises were constructed to improve the endurance and physical strength of the adolescents.

All interventions were conducted by experts from the EDDY study team. The lifestyle intervention was held by physicians. All nutritional training lessons were developed and conducted by nutritionists, and the sports and movement intervention was planned and held by sports scientists. The team was supported by psychologists to ensure a sensitive and age-appropriate implementation of the interventions.

\section{Statistical Analysis}

Statistical analysis was performed using SPSS 14 (IBM, Armonk, NY, USA). Descriptive analysis was conducted to classify the sample according to BMI z score, BMI percentiles, and body fat percentage at baseline.

Changes in the nutritional knowledge and nutritional behavior as well as gender comparisons were measured using t-test. All variables were tested for normal distribution prior to analysis. As data on body fat were skewed, nonparametric tests were applied. Statistical tests were two-sided. P values smaller than 0.05 were considered statistically significant.

\section{Results}

\section{Body Fat Percentage}

At baseline the sample $(n=115)$ showed a mean value of $23.5 \pm 7.1 \%$ body fat. This proportion is classified in the upper third of the normal range, defined by the 50th percentile 
Table 2. Frequency of overweight and underweight according to body fat percentile curves of McCarthy et al. [4]

\begin{tabular}{lll}
\hline & Frequency & \% Amount of study population \\
\hline Underweight $(<2$ nd percentile) & 4 & 3.5 \\
Normal weight (2nd to 85th percentile) & 76 & 0 \\
Overweight $(>85$ th percentile) & 19 & 16.5 \\
Obese ( $>$ 95th percentile) & 16 & 14.0 \\
\cline { 2 - 3 } Total & 115 & $100 \%$ \\
\hline
\end{tabular}

according to Mc Carthy et al. [4]. The mean body fat percentage was $23.1 \pm 8.6 \%$ in the male subjects $(n=57)$ and $23.9 \pm 5.4 \%$ in the female subjects $(n=58)$.

At the time of the first data collection, $66 \%$ of the pupils were located between the 2 nd and 85th body fat percentile according to Mc Carthy et al. [4]. Therefore, nearly two-thirds of the study population can be considered as normal according to their body fat percentage. Significant to pathological values can be localized both in the upper and lower weight segments. According to the $\%$ body fat percentile curve, $3.5 \%$ of the pupils are underweight, $16.5 \%$ are classified as overweight and $14 \%$ as obese. Thus, $30.5 \%$ of the pupils are classified as overweight according to the percentile curves of McCarthy et al. [4] (table 2).

At baseline, the subjects of the control group had a lower body fat percentage than those assigned to the intervention group. In the first follow-up measurement after 6 months and participation of the first intervention cycle, no significant difference between control and intervention group could be identified. Similarly, the two groups did not differ significantly in terms of their body fat percentage after participation in the second intervention cycle, but it was noted that the subjects of the intervention group had a lower increase regarding their body fat percentage than the participants assigned to the control group; however, the difference was not significant ( $n=115 ; p=0.095$ ) (fig. 2).

The mean z-score of the intervention group and the control group at baseline was $0.62 \pm$ 1.28 and $0.28 \pm 1.09$, respectively $(p=0.96)$. After intervention, the mean $z$-score of the intervention group was $0.41 \pm 1.32$ compared to $0.58 \pm 1.21$ in the control group ( $p=0.97)$.

\section{Nutritional Knowledge}

Before intervention, the subjects were able to answer an average of $40 \%(n=79)$ of the 12 questions posed correctly.

The comparison of knowledge before and after 6 months of intervention shows an increase in knowledge by an average of $12.4 \%$ immediately after intervention compared to the baseline levels and a further increase of $9.3 \%$ at the last follow-up. Overall, the nutritional knowledge after intervention has improved by an average of $21.6 \%$ compared to the baseline survey before the start of intervention, which corresponds to a significant increase in nutritional knowledge $(n=79 ; p<0.001)$ (fig. 3 ).

\section{Nutritional Habits}

The comparison of selected foods shows a significant reduction in the consumption of the following food groups before and after intervention. The weekly consumption of fast food products has reduced significantly from median (Med) $=3$ before the beginning of the intervention to Med $=2$ at the last follow-up, as it was true for the consumption of sweets ( $\mathrm{n}=81$; $\mathrm{p}<0.001$; Med before intervention $=2$; Med at last follow-up $=1)$, salty snacks $(\mathrm{n}=81 ; \mathrm{p}<$ 0.001; Med before intervention $=2$; Med at last intervention $=1)$, and instant meals $(n=81$; $\mathrm{p}<0.001$, Med before intervention = 2; Med at last follow-up = 1,5) (fig. 4). 
Widhalm et al.: The Viennese EDDY Study as a Role Model for Obesity: Prevention by Means of Nutritional and Lifestyle Interventions

Fig. 2. Body fat percentage before and after intervention.

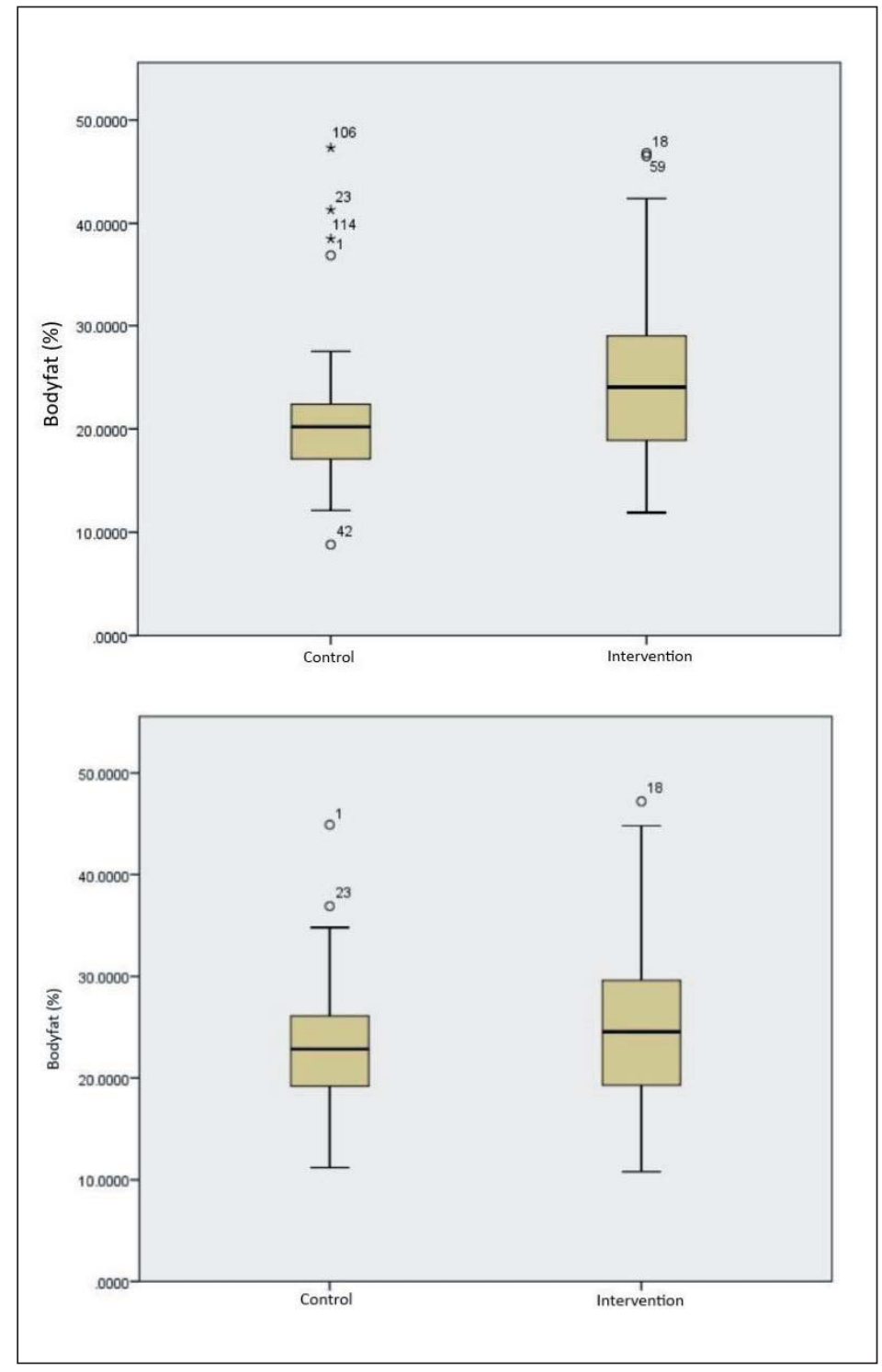

\section{Physical Fitness}

No significant improvements in the physical fitness could be identified. However, the jumping distance of the adolescents participating in the intervention improved by $10.3 \mathrm{~cm}$ after the first intervention cycle. The range measured in the sit-and-reach test also increased by $4.3 \mathrm{~cm}$. In addition, the endurance performance improved by $23 \mathrm{~m}$. These effects were not retained after the second intervention cycle.

\section{Psychology}

At baseline the subjects had a low motivation for physical activity $(n=81 ; m=47.2)$ as well as a high score in uncontrolled and unhealthy eating habits $(\mathrm{n}=81 ; \mathrm{m}=53.5)$ and preclinical eating disorders $(n=81 ; m=55.6)$. After the final intervention the motivation for movement increased in a non-significant extent $(\mathrm{n}=81 ; \mathrm{m}=49.1)$. A non-significant impact on the eating habits could have been shown $(n=81 ; m=50.7)$ as well as a significant impact on pre-clinical eating disorders $(n=81 ; m=49.7 ; p=0.004)$. 
Widhalm et al.: The Viennese EDDY Study as a Role Model for Obesity: Prevention by Means of Nutritional and Lifestyle Interventions

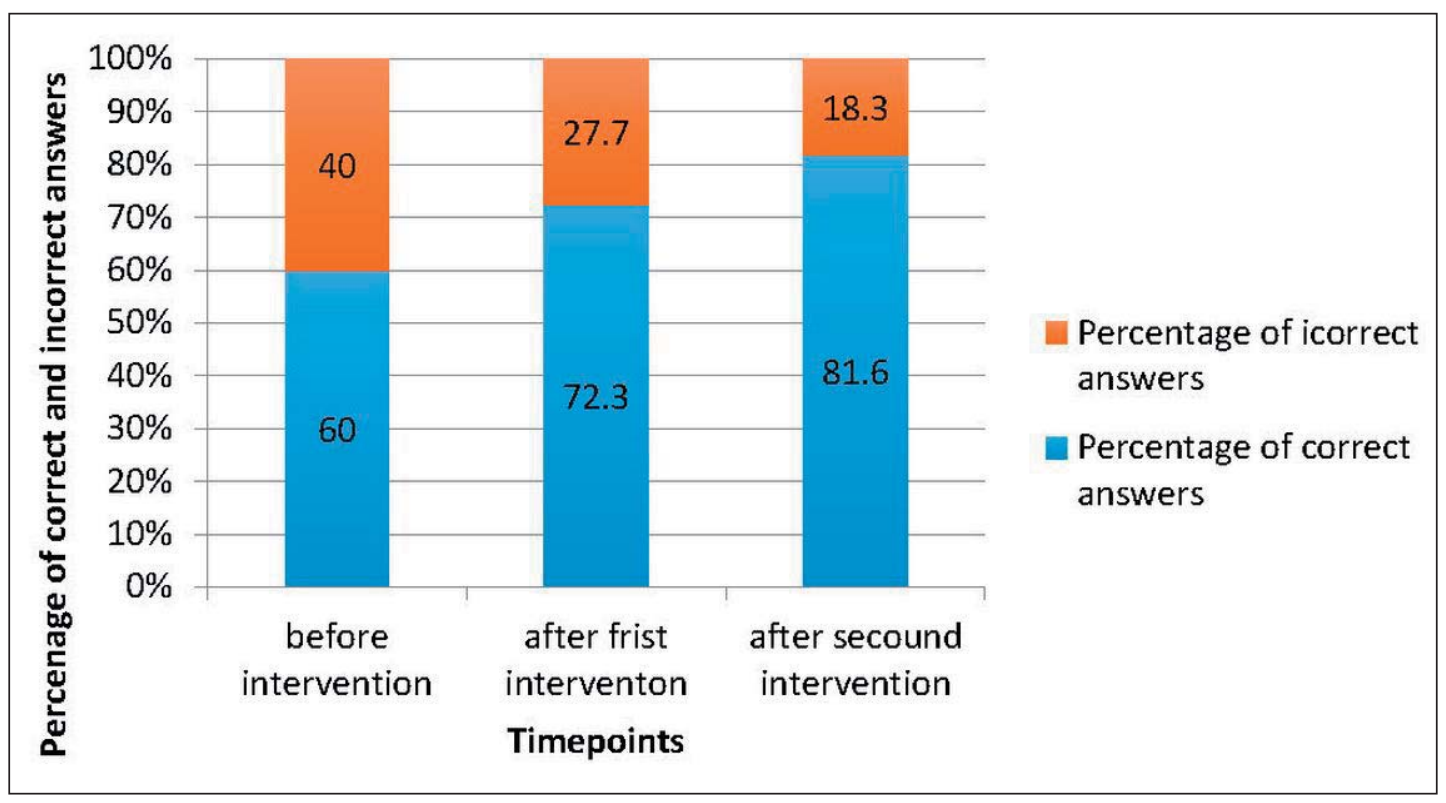

Fig. 3. Nutritional knowledge.

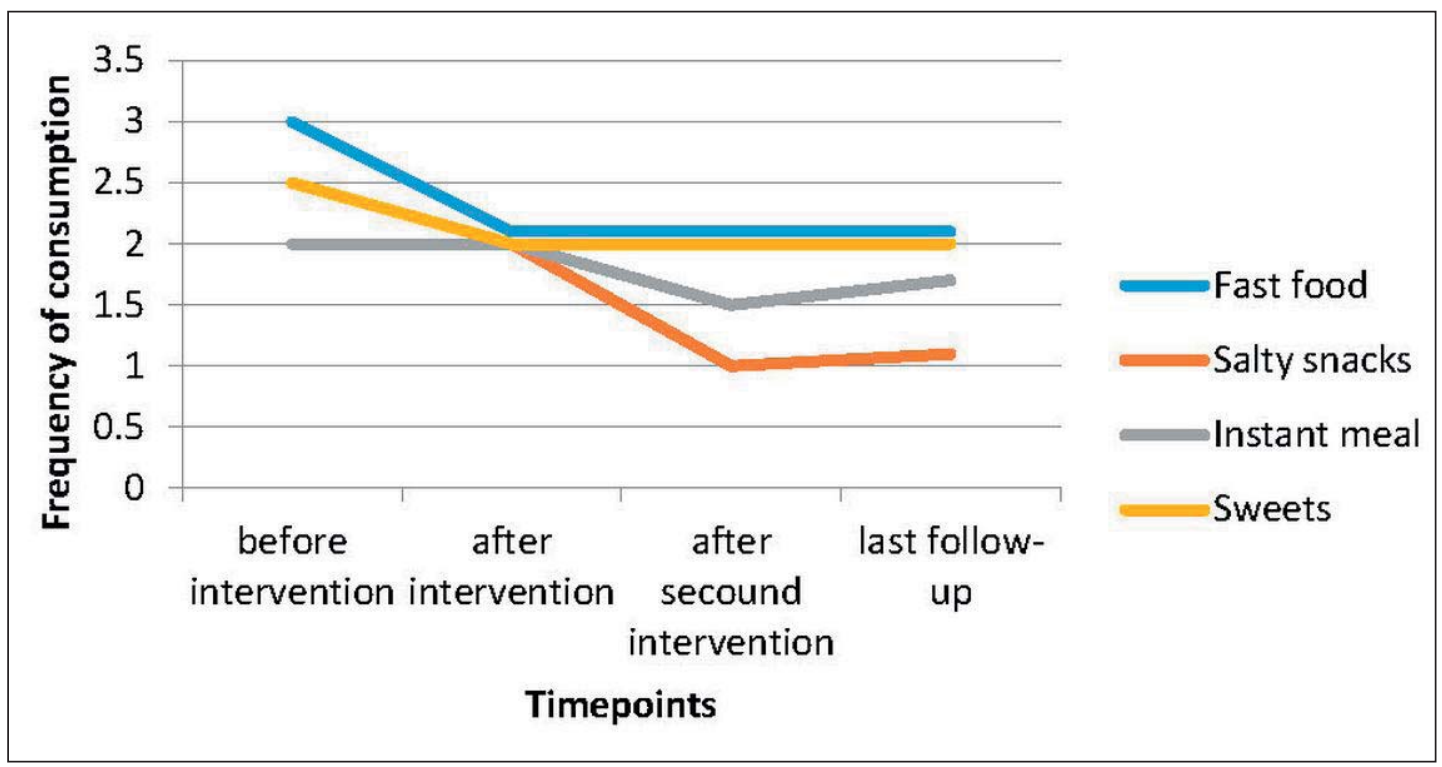

Fig. 4. Nutritional habits.

\section{Discussion}

The study at hand was conducted as a school-based intervention study in adolescents. We provide evidence that a combined sports and nutritional intervention with a duration of 1 year can significantly improve the eating habits and the nutritional knowledge of Viennese school children. Corresponding with these changes, a non-significant improvement of the body fat percentage as well as the physical performance was identified. 
Previous data describe the effects of preventive interventions on overweight and cardiovascular risk factors in children and adolescents. The main influencing factors were identified as combined nutritional and movement interventions, which improve the health behavior of children and adolescents and reduce the risk of developing overweight and its consequences by improving their nutritional knowledge and physical activity [10-15]. Most programs focus on behavioral prevention and school-based interventions, which were most effective in direct communication with the adolescents [16]. An improvement of the weight status in overweight children and adolescents has also been shown by combined physical exercise and nutrition education and behavior therapy [17], the effects of which were ongoing after a 1-year follow-up [18].

According to the multifactorial approach to improve positive health, the consideration of psychological factors is emphasized in addition to physical and social factors [19].

Therefore, psychological factors are important aspects of prevention measures. The psychological data of the EDDY study underline the importance of this dimension and point out that this third dimension of positive health development will be considered even more in the future and that pupils could benefit from psychological interventions. In addition, the results point out the abnormal eating habits and motivation for movement.

One key factor of health promotion measures is the improvement of knowledge in the forced intervention area through specific intervention. Nutritional knowledge of the subjects was measured through their ability to correctly answer a 12-question quiz about nutrition. The nutritional knowledge of the subjects improved significantly after participation in the intervention, which indicates that the intervention was effective in regard to educational factors.

Our data shows an increase of the body fat percentage after participation in the nutrition and exercise training in a statistically non-significant extent. It is important to note that the mean body fat percentage was higher in the intervention group at baseline compared to the mean body fat percentage of the control group. At the conclusion of the study, however, the intervention group showed lower body fat percentages than the control group, whereas the subjects of the intervention group had a lower body fat percentage compared to those who did not participate in the intervention.

Another factor for measuring the health status are the eating habits of the subjects. In addition to the educational effectiveness, it is an important target of prevention measures to evaluate the efficiency of a lifestyle intervention. The pre-post comparison of the consumption of fast food, instant meals, sweets, and salty snacks in the EDDY subjects shows a significant reduction in the consumption of all four food groups after completion of the intervention program.

Although both cohorts were comparable regarding their body fat percentage at the time of recruitment, a number of dropouts occurred in the control group. Thus, in the per protocol analysis the baseline values for body fat in the control group were significantly lower than that of the intervention group. However, over the course of the study an increase in body fat occurred in the control group while the body fat of the intervention group remained unchanged. This effect could suggest that the intervention was successful in preventing a further increase of body fat.

Changes in both variables, the body fat percentage and eating habits, in the subjects of the EDDY study imply an impact of the intervention on the subject's lifestyle.

The EDDY study has several strengths and limitations. The main weakness of the study is its relatively small number of subjects. Furthermore, the study population consists of Viennese adolescents and the data may not be representative for the Austrian population in total. In addition, puberty in girls occurs earlier than in boys of the same age. These differences can disrupt the comparison of sexes in adolescents, especially in the comparison of anthropometric data, but also for metabolic measurements and psychological data. 
Strengths of the EDDY study are a randomly selected study population with generally healthy school children, which nevertheless show a very large variation in their BMI and body fat percentage. Particularly the evaluation of blood samples from unselected healthy young people is noteworthy and may be helpful to identify risk populations for cardiovascular and metabolic disease in adolescence.

Overall, our results suggest that an improvement in nutritional knowledge through combined nutritional and exercise training within a school-based prevention program can improve both the health behavior and the health status of the subjects. These data complement the knowledge of a few recent, controlled prevention studies, which describe a benefit of dietary and exercise training on the health of pupils, and show evidence that an age-appropriate dietary and exercise intervention can have a benefit on the lifestyle of adolescents.

\section{Acknowledgments}

We thank the pupils participating in this project for their outstanding cooperation. Furthermore, we want to thank the schools as well as the parents and legal guardians for their support.

We also thank the co-workers of the EDDY study (Mrs. Christina Pöppelmeyer etc.) for their excellent work and engagement.

The EDDY Study was supported by the Austrian Heart Foundation, Vienna, Austria; the Hofer KG, Sattledt, Austria; Nestlé Österreich GmbH, Vienna, Austria.

\section{Disclosure Statement}

We declare no conflict of interest.

\section{References}

1 Report of the Commission on Ending Childhood Obesity: Implementation Plan, 2017 Executive Board, 140th Session, Provisional Agenda intem 10.4, s.L

-2 NCD Risk Factor Collaboration (NCD-RisC), Worldwide trends in body-mass index, underweight, overweight, and obesity from 1975 to 2016: a pooled analysis of 2416 population-based measurement studies in $128 \cdot 9$ million children, adolescents, and adults. Lancet 2017;390:2627-2642.

-3 Béghin L, Huybrechts I, Vicente-Rodríguez G, De Henauw S, Gottrand F, Gonzales-Gross M, Dallongeville J, Sjöström M, Leclercq C, Dietrich S, Castillo M, Plada M, Molnar D, Kersting M, Gilbert CC, Moreno LA: Main characteristics and participation rate of European adolescents included in the HELENA study. Arch Public Health 2012;70:14.

4 Mc Carthy HD, Jole TJ, Fry T, Jebb SA, Prentice AM: Body fat reference curves for children. Int J Obes 2006;30: 598-602.

5 Must A, Anderson SE: Body mass index in children and adolescents: considerations for population-based applications. Int J Obes 2006;30:590-594.

-6 Poeppelmeyer C, Helk O, Mehany S, Hauer R, Pachinger O, Widhalm K: Die Wiener Präventionsstudie EDDY - erste Ergebnisse. Pädiatr Pädol 2016;51:104-108.

7 Truthman J, Mensik, G, Richter A: Relative validation of the KiGGS Food Frequency Questionnaire among adolescents in Germany. Nutr J 2011;10:133.

8 Bös K, Schlenker L, Büsch L, Lämmle L, Müller H, Oberger J, Seidel I, Tittlbach S: Deutscher Motorik Test 6-18 (DMT 6-18). Hamburg, Czwalina, 2009.

9 Ardelt-Gattringer E, Meindl M: Interdisziplinäres Testsystem zur Adipositas- Diganostik und Evaluation bei Adipositas und anderen durch Ess- und Bewegungsverhalten beeinflussbare Erkrankungen. Bern, Huber, 2010.

-10 Jouret B, Ahluwalia N, Dubuy M, Cristini C, Negre-Pages L, Grandjean H, Tauber M: Prevention of overweight in preschool children: results of kindergarten-based interventions. Int J Obes 2009;33:1075-1083.

$\checkmark 11$ Taylor RW, Mcauley KA, Barbezat W, Strong A, Williams SM, Mann JI: APPLE Project : 2-y findings of a community-based obesity prevention program in primary school-age children. Am J Clin Nutr 2007;86:735742.

12 Yin Z, Moore J, Johnson MH, Vernon M, Gutin B: The Impact of a 3-Year after-school obesity program in elementary school children. Child Obes 2012;8:60-70. 
Widhalm et al.: The Viennese EDDY Study as a Role Model for Obesity: Prevention by Means of Nutritional and Lifestyle Interventions

13 Skybo TA, Ryan-Wenger N: A school-based intervention to teach third grade children about the prevention of heart disease. Pediatr Nurs 2002;28:223-229, 235

14 Sahota P, Rudolf MJ, Dixey R, Hill AJ, Barth JH, Cade J: Randomised controlled trial of primary school based intervention to reduce risk factors for obesity. BMJ 2001;323:1029.

15 Kain J, Uauy R, Albala C, Vio F, Cerda R, Leyton B: School-based obesity prevention in Chilean primary school children: methodology and evaluation of a controlled study. Int J Obes 2004;28:483-493.

-16 Blüher S, Kromeyer-Hauschild K, Graf C, Grünewald-Funk D, Widhalm K, Korsten-Reck U, Markert J, Güssfeld C, Müller MJ, Moss A, Wabitsch M, Wiegand S: Current guidelines to prevent obesity in childhood and adolescence. Klin Paediatr 2015;228:1-10.

17 Schaefer A, Winkel K, Finne E, Kolip P, Reinehr T: An effective lifestyle intervention in overweight children: one-year follow-up after the randomized controlled trial on 'Obeldicks light'. Clin Nutr 2011;30:629-633.

18 Reinehr T, Schaefer A, Winkel K, Finne E, Toschke AM, Kolip P: An effective lifestyle intervention in overweight children: findings from a randomized controlled trial on 'Obeldicks light'. Clin Nutr 2010;29:331-336.

19 Pelikan JM: Ausdifferenzierung von spezifischen Funktionssystemen für Krankenbehandlung und Gesundheitsförderung oder: Leben wir in der «Gesundheitsgesellschaft»? Österreichische Zeitschrift für Soziologie 2009;34:28-47. 